\title{
CORRESPONDENCE
}

\section{Physical health in mental illness: psychiatry's shared responsibility}

I have recently completed the evaluation of responses to a postal questionnaire sent to consultants in adult general psychiatry in the UK. The aim was to study their understanding, recognition and management of ill health in patients with severe mental illness. The 300 responses ( $72 \%$ response rate) received have been helpful in revealing the present state of practice in recognising the problems and addressing the unmet health needs of this patient group.

Only $72 \%$ of the consultants who responded thought that patients have a complete physical examination on admission to an acute psychiatric ward; $28 \%$ believed that patients do not have a full physical examination within $72 \mathrm{~h}$ of admission.

As regards out-patients, $26 \%$ of consultants believed that the medical needs of their patients are managed by general practitioners (GPs) via care coordinators. Only $45 \%$ reported that their patients had been asked to have physical check-ups at GP surgeries in the previous 3 years. The rest did not know whether their patients have any medical needs nor whether their needs are addressed at primary care level.

Fifty-six per cent of respondents felt that the physical health of out-patients, albeit important, is the responsibility of primary care. As psychiatrists, they did not get involved in investigating possible medical conditions of their patients and they did not expect to receive information from primary care. Of the $44 \%$ who thought they should be involved in their patients' well-being, 51\% reported that they have minimal time or resources to deal with physical health questions; $45 \%$ said that they have moderate resources and could mobilise these if the need arose; only $4 \%$ reported that they have provisions to address the physical needs of their patients.

I therefore welcome recent articles in APT focusing on the physical health and lifestyle of people with severe mental illness and problems of their management (Connolly \& Kelly, 2005; Garden, 2005; Lester, 2005). It is important to draw colleagues' attention to (belated) protocols of shared care for people with long-standing mental health problems.

Training for all mental health professionals working with people with severe mental illness and the increased drug-related risk of weight gain, hypercholesterolaemia, hyperprolactinaemia, diabetes, metabolic syndrome and other conditions affecting patients will need to come to the forefront of concerns when assessing the quality of mental health provision for this patient group.

Connolly, M. \& Kelly, C. (2005) Lifestyle and physical health in schizophrenia. Advances in Psychiatric Treatment, 11, 125132

Garden, G. (2005) Physical examination in psychiatric practice. Advances in Psychiatric Treatment, 11, 142-149.

Lester, H. (2005) Shared care for people with mental illness: a GP's perspective. Advances in Psychiatric Treatment, 11, 133141.

Dora Kohen Consultant Psychiatrist, Lancashire Postgraduate School of Medicine, Preston, UK. E-mail: dorakohen@ doctors.org.uk

\section{Smoking in a long-stay psychiatric rehabilitation centre}

We read with interest the comprehensive article on lifestyle and physical health in schizophrenia by Connolly \& Kelly (2005). Physical health problems in chronic mental illness are recognised causes of morbidity and mortality (Brown et al, 1999). Previous studies have reported a very high prevalence of smoking (75-92\%) in people with psychotic disorders (Kelly \& McCreadie, 2000).

In a survey of two long-stay psychiatric rehabilitation wards in Lincoln, 31 patients (21 men, 10 women; mean age $=43$ years; s.d. $=10 ; 27 / 31$ with a diagnosis of schizophrenia) were asked to complete the short version of Fagerstrom questionnaire (Heatherton et al, 1991). This is a highly reliable and valid questionnaire widely used to measure levels of nicotine dependence. The higher the score, the greater the likelihood of dependence and of benefit from nicotine replacement therapy.

Of the 31 participants, $24(77.4 \%)$ were smokers, more than half of whom were either highly or very highly dependent on nicotine $(11.5 \%$ very highly dependent, $42 \%$ highly dependent, $11.5 \%$ moderately and $12.4 \%$ mildly dependent). About two-thirds $(62 \%)$ of the participants were overweight and onethird had comorbid physical illnesses such as diabetes, asthma or thyroid disorder.

Reducing smoking rates in people with schizophrenia, together with better management of physical illness, are suggested strategies to reduce high mortality rates (Cormac et al, 2005). By adopting a shared care model, mental health professionals with the help of primary care services could effectively address the problem of unhealthy life styles in people with mental illness. 
Sports facilities should be available to engage patients in physical activities. Dietary advice by a hospital nutritionist and provision of a healthy diet would be extremely useful in improving the physical health of the long-stay patients. In addition, nicotine dependence can be tackled through liaison with primary care teams offering education, support and nicotine replacement therapy.

Brown, S., Birtwistle, J., Roe, L., et al (1999) The unhealthy lifestyle of people with schizophrenia. Psychological Medicine, 29, 697-701.

Connolly, M. \& Kelly, C. (2005) Lifestyle and physical health in schizophrenia. Advances in Psychiatric Treatment, 11, 125132.
Cormac, M., Ferriter, R., Benning, R., et al (2005) Physical health and health risk factors in a population of longstay psychiatric patients. Psychiatric Bulletin, 29, 1820.

Heatherton, T. F., Kozlowski, L. T., Frecker, R. C., et al (1991) The Fagerstrom Test for Nicotine Dependence: a revision of the Fagerstrom Tolerance Questionnaire. British Journal of Addiction, 86, 1119-1127.

Kelly, C. \& McCreadie, R. (2000) Cigarette smoking and schizophrenia. Advances in Psychiatric Treatment, 6, 327331.

Reza Kiani Senior House Officer in Psychiatry, Lincolnshire Partnership NHS Trust, UK. E-mail: reza.kiani@lpt.nhs.uk

Mohammed Abbas Senior House Officer in Psychiatry, Lincolnshire Partnership NHS Trust

\section{APT eLetters}

Aggravated by an article in APT? - Why not tell us?

\section{You can comment immediately on APTs content}

The eLetters section featured in the online APT is a quick and easy method for you to submit a rapid response to any article.

Accepted letters are posted online within 7 days of your submitting them. The Editor moderates all correspondence before it is posted on the site, where it can be accessed through a general link or directly from the article to which it refers. All eLetters are considered for subsequent publication in the print correspondence column.

Try for yourself from an online full-text article.

http://apt.rcpsych.org

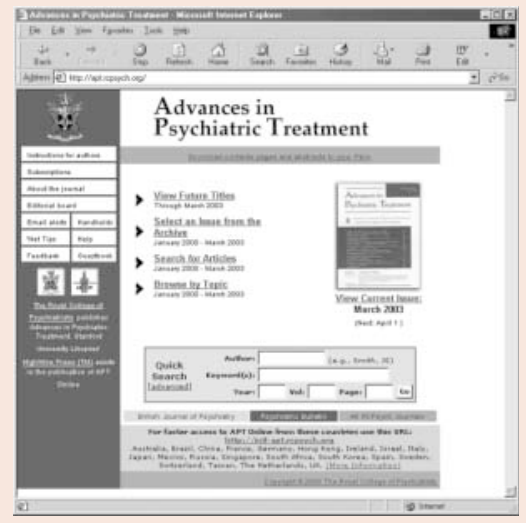

